\title{
Transient visually evoked potentials to sinusoidal gratings in optic neuritis
}

\author{
GORDON T PLANT \\ From the Department of Neurology, Addenbrooke's Hospital, and the Physiological Laboratory, Cambridge \\ $U K$
}

SUMMARY Transient visually evoked potentials (VEPs) to sinusoidal gratings over a range of spatial frequencies have been recorded in cases of optic neuritis. The use of the response to pattern onset in addition to the response to pattern reversal extended the range to higher spatial frequencies by up to two octaves. There was an increase in VEP delay and a greater degree of discrimination from a control group at higher spatial frequencies. This finding is discussed in the light of previous reports of luminance and checkerboard VEPs in demyelinating optic nerve disease. An attempt is made to relate amplitude changes in various VEP components to contrast sensitivity measurements in this group of patients.

Following the demonstration by Halliday $e$ al $^{1}$ of the delayed pattern visually evoked potential (VEP) in optic neuritis and multiple sclerosis ${ }^{2}$ the test has become a useful clinical tool in the diagnosis of the latter condition. The earliest reports, however, of more than a $90 \%$ incidence of abnormalities in the important "probable" and "possible" diagnostic categories have not been confirmed by subsequent workers who have found abnormalities in only, for example, $19 \%^{3}$ or $38 \%$ of the "possible" group. This has prompted attempts to improve the diagnostic yield by modifying the parameters of the visual stimulus. Change in luminance ${ }^{5}$ and orientation ${ }^{6} 7$ has been tried, as has the use of additional stimuli such as a foveal stimulus ${ }^{8}$ or photic driving, ${ }^{9}$ each with some success.

The final objective of the present study is similar. Rather than study a large number of patients referred for diagnosis it was proposed to investigate a small number of patients known to have optic neuritis and to look at each in greater detail than would otherwise be practicable. The stimulus parameter that has been varied is the size of the pattern elements. In preference to changing the check size of the more commonly used checkerboard stimulus, gratings with a sinusoidally varying luminance profile have been employed and their spatial fre-

Address for reprint requests: Dr GT Plant, Addenbrooke's Hospital, Hills Road, Cambridge, CB2 2QQ, UK.

Received 12 March 1983 and in revised form 15 June 1983. Accepted 28 June 1983. quency (periodicity of the light and dark bars of the grating) altered. Following the introduction of such gratings by Schade ${ }^{10}$ and the suggestion by Campbell and Robson ${ }^{11}$ of their potential significance to visual processing there has been much experimental work establishing their importance in the study of the visual system in health and disease. In the present context a cogent argument for the use of gratings as opposed to checks is that the former may be a more selective means of stimulating neural elements sharing a particular receptive field size. It is this that is hoped to be achieved by varying the spatial periodicity of the stimulus.

\section{Method}

Vertical gratings with sinusoidally varying luminance profiles were generated on an oscilloscope screen $\left(\mathbf{P}_{31}\right.$ phosphor). The space-averaged luminance of the screen was $200 \mathrm{~cd} / \mathrm{m}^{2}$ which remained constant in time however the pattern on the screen was modulated. The contrast of the gratings used was at all times $55 \%$. The screen was masked to give a circular field and the surround illuminated to approximately the same luminance and hue as the screen. In pattern reversal mode the grating was counterphase shifted in square-wave fashion at $1 \mathrm{~Hz}$ (that is the bright and dark bars abruptly exchanged position at $500 \mathrm{~ms}$ intervals). The signal effecting the pattern reversal also triggered the averager. In pattern onset mode the grating was alternated with a blank field of the same average luminance at $1 \mathrm{~Hz}$ but the averager was only triggered when the grating was turned on. Silver-silver chloride electrodes were used, the active electrode being in the mid-line $5 \mathrm{~cm}$ above the inion referenced to an electrode situated at 
the vertex. The earth electrode was also in the mid-line equidistant between the other two. Potentials were conventionally amplified using a band-width of $1 \cdot 6-80 \mathrm{~Hz}$. The single channel signal was averaged by means of a mini-computer (CA alpha) incorporated in an Evoked Response System (Cambridge Electronic Design). Twohundred and fifty-six responses were averaged throughout and the sampling frequency was $800 \mathrm{~Hz}$ with a time-base of $300 \mathrm{~ms}$. The results were stored on floppy disc for subsequent analysis.

Monocular VEPs with natural pupils were recorded in all instances. The ambient illumination in the room was kept at a constant, low level. The field size and viewing distance were varied according to the desired spatial frequency of the stimulus as gratings finer than 4 cycles $/ \mathrm{cm}$ on the screen could not be generated. For spatial frequencies of 2 cycles/degree $\left(\mathrm{c}^{\circ}\right)$ and below the viewing distance was $57 \mathrm{~cm}$ and the screen subtended $20^{\circ}$ of visual angle. Up to $10 \mathrm{c}^{\circ}$ the viewing distance was increased to $114 \mathrm{~cm}$ giving a field size of $10^{\circ}$ and higher spatial frequencies were recorded at $228 \mathrm{~cm}$. A fixation mark was provided to stabilise accommodation and minimise eye movements. Contrast sensitivities were measured later the same day using a separate but identical display. Stationary gratings were used and the patient varied the contrast of the stimulus himself using a logarithmic attenuator, gratings being presented initially below threshold and the contrast increased until they were deemed just visible. The mean of at least three settings was always taken.

\section{Subjects}

Seven control subjects, having a similar age distribution to the patients, were used. The thirteen patients were selected according to the following criteria: (1) All had had an attack of unilateral optic neuritis more than 2 and less than 18 months previously. (2) All had modest, if any, residual deficit as judged by symptomatology, Snellen acuity and Ishihara testing. (3) Where patients had had checkerboard VEPs they were known to have modest abnormalities of latency and amplitude. (4) None had any previous history of visual or other neurological dysfunction. (5) None had central or paracentral scotomata detectable by conventional static perimetric techniques. All patients and controls wore corrective lenses appropriate to the viewing distance where necessary.

\section{VEP analysis}

Peak latencies were measured. The amplitude of the first major negative wave (called N1) was measured either from its take-off or from the peak of the preceding positive wave if present. The amplitude of the first major positive component (P1) was measured from the $\mathrm{N} 1$ peak and that of the second major positive wave (P2) from the preceding negative peak.

\section{Results}

Figure 1 shows computer print-outs of VEPs recorded in three of the patients studied. It will be noted that a double peak to the major positive wave occurs at the lowest spatial frequencies in one sub- ject. Pattern reversal responses are dominated by the major positive wave, P1; and pattern onset responses by the major negative wave, N1. These findings in normal subjects have been discussed in detail elsewhere. ${ }^{12}$

In fig 2 VEP amplitudes at different spatial frequencies in four of the patients are plotted. The amplitude of the pattern reversal potential above $4 \mathrm{c}^{\circ}$ diminishes rapidly whereas the amplitude of the pattern onset response peaks at a higher spatial frequency. In the present study, therefore, pattern reversal responses were utilised up to, and pattern onset responses beyond $4 \mathrm{c} /{ }^{\circ}$. In 11 patients whose visual acuity was $6 / 9$ or better usable VEPs were obtained up to 14 or $16 \mathrm{c}^{\circ}$ in the affected eyes. In two patients whose Snellen acuity was $6 / 18$ reliable VEPs were not obtained in pattern onset mode, either because of low amplitude or very abnormal waveform. This is considered to reflect the greater selectivity of the pattern onset response to the presence of fine detail within the stimulus.

Figure 3 shows a comparison of the VEP latencies in the affected eyes of the patient group with the latencies obtained in the control subjects. P1 latency was measured in the case of pattern reversal responses and $\mathrm{N} 1$ latency in the case of pattern onset. Mean latencies $( \pm \mathrm{SD})$ obtained in the 11 patients in whom VEPs were recorded across the entire spatial frequency spectrum are shown.

It was found that the difference in latency between the normal and abnormal groups varied in a consistent way with change in spatial frequency. In part this may reflect the increase in absolute latency seen with increasing spatial frequency: as if the added delay is proportional to the normal latency. This cannot be the entire explanation, however. Below $1 \mathrm{c} /{ }^{\circ}$ the two groups cease to differ significantly despite the fact that the mean latency in the normal group is greater at $0.25 \mathrm{c}^{\circ}$ than at $1 \mathrm{c} / 0$. Furthermore, when the stimulus mode switches to pattern onset the mean delay at $4 \mathrm{c} /{ }^{\circ}$ is $14 \mathrm{~ms}$, where the mean latency in the normal group is $73 \mathrm{~ms}$. This is the same mean delay as seen in the $2 \mathrm{c} /{ }^{\circ}$ pattern reversal condition where the expected latency is 93 ms.

In fig 4 the same data are plotted in a different way. It is known that the interocular difference in latency shows less variability in normals than does the absolute latency. It may therefore achieve better discrimination between normal and abnormal. The interocular latency difference is shown in each of the patients studied for each of the spatial frequencies at which reliable responses were obtained. The dashed line gives the interocular difference in the normal group \pm 3 SD. The trend shown in fig 3 is again seen but only at the highest spatial frequency are all the 


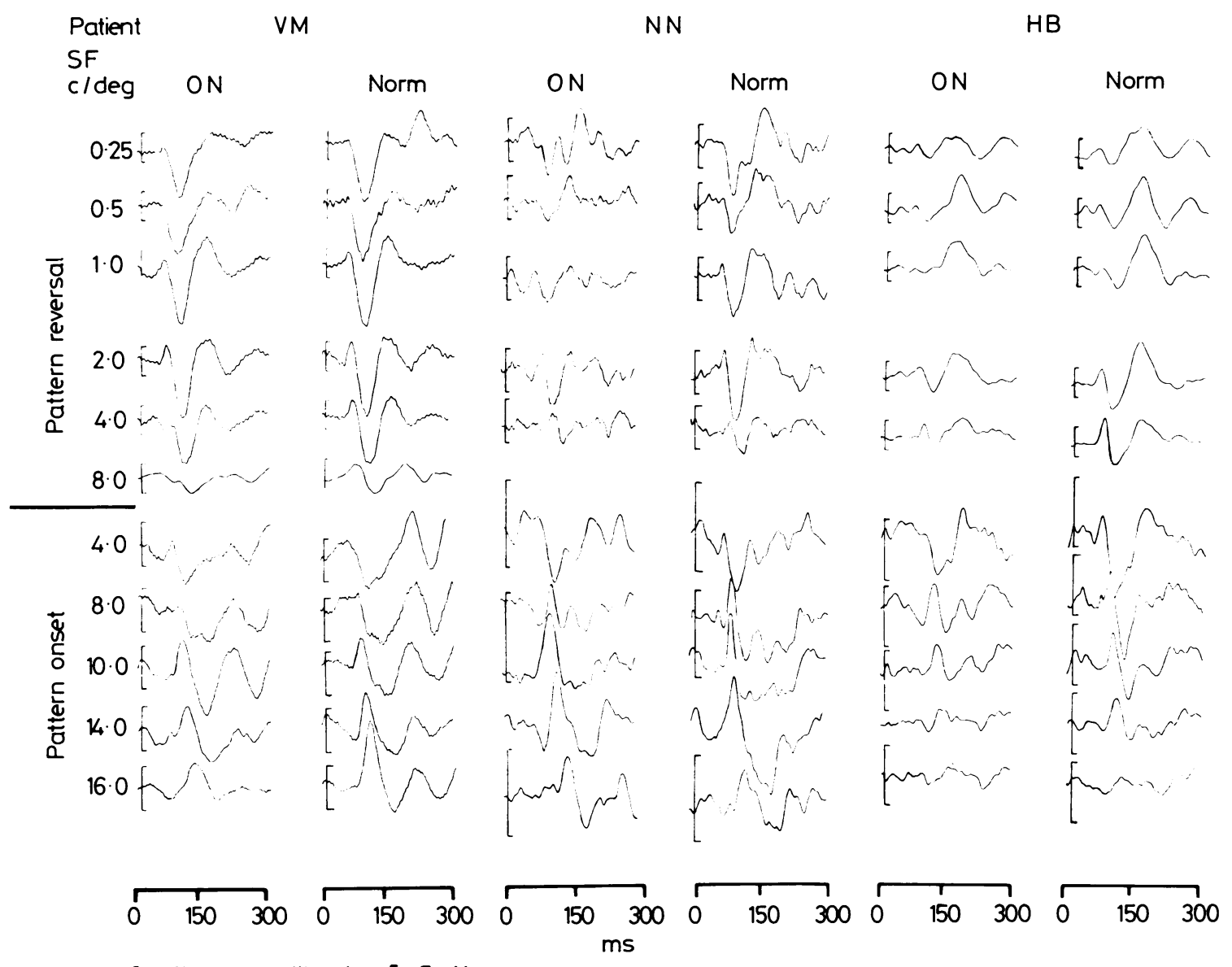

In all cases calibration $[=5 \mu \mathrm{V}$

Fig 1 Computer print-outs of families of VEPs obtained to gratings of different spatial frequency in three of the cases of unilateral optic neuritis $(O N)$. Positivity is represented by a downward deflection. The importance of the first major positive component in pattern reversal and the negative component in pattern onset responses is seen. There is great variability of waveform below $1 \mathrm{c}^{\circ}$ and of the later waves throughout.

patients outside the normal range when it is defined in this way.

An attempt was made to relate amplitude changes in the VEPs to threshold contrast sensitivity deficits at the same spatial frequency. The ratio of the amplitude in the affected and unaffected eyes was used in this analysis in view of the great variation in amplitude between individuals and across spatial frequency. The contrast sensitivity loss was taken as the difference in threshold contrast sensitivity between the affected and unaffected eyes in DB.

In fig 5 the amplitude ratios are plotted against the contrast sensitivity deficits at the same spatial frequencies for the $\mathrm{N} 1, \mathrm{~N} 1-\mathrm{P} 1$ and $\mathrm{N} 2-\mathrm{P} 2$ components. Pattern reversal responses to gratings of $1 \mathrm{c}^{\circ}$ and below have been plotted separately as have pattern onset VEP results. Regression lines have been fitted by the method of least squares. As can be seen the strongest correlation was found for the N1-P1 component above $1 \mathrm{c}^{\circ}$. This finding may in part be due to the fact that the N1-P1 amplitude is the most robust measurement. It is little influenced by variations in the baseline unlike the $\mathrm{N} 1$ amplitude when measured from take-off, as was necessary in the majority of patients. The N2-P2 amplitude is notorious for showing greater variability and indeed in the present group of patients could not be identified at all in a significant proportion, particularly in pattern onset responses. Below $1 \mathrm{c}^{\circ}$ all of the components measured show greater variability 


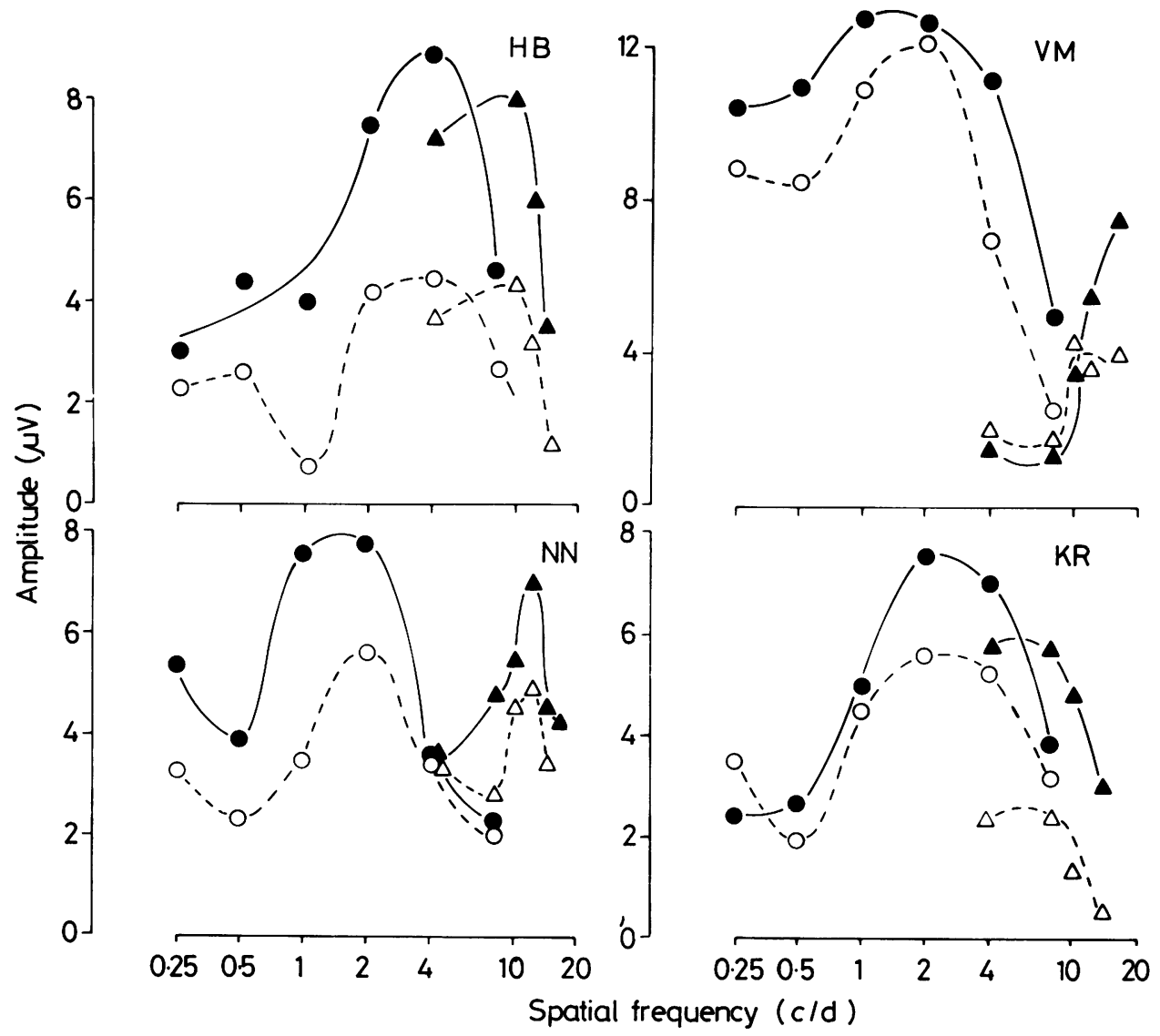

Fig 2 VEP amplitude is plotted as a function of spatial frequency in four of the patients studied. Filled symbols are unaffected eye and unfiled symbols affected eye results. It should be noted that the pattern reversal response $(0,0)$ encompasses a lower spatial frequency range than does the pattern onset response $(\Delta, \Delta) . N 1-P 1$ amplitude has been plotted throughout except for the pattern onset result for patients VM and NN. In these cases the NI amplitude is shown to demonstrate that this component has a higher spatial frequency selectivity because it is an insignificant component of medium spatial frequency $V E P s$.

because of the variation in wave-form, both between subjects and between the two eyes in the same subject.

\section{Discussion}

The overall pattern of wave-form, amplitude and latency variation with change in the spatial frequency of the stimulus seen in the present group of patients and controls is in accord with that reported from this laboratory in a larger group of normal subjects. ${ }^{12}$

As stated above the aim of this study was to determine whether varying the spatial properties of the stimulus had any effect on the discriminatory properties of VEPs in demyelinating optic nerve disease. There are reasons to suppose that employing sinusoidal gratings may be a more satisfactory means of exploring this possibility than the use of checks. Recent neurophysiological experiments have demonstrated that whereas visual cortical cells have a narrow response range to gratings of different spatial frequencies ${ }^{13}$ a checkerboard stimulus will excite populations of cells each responding to spatial properties of the stimulus which can be predicted from a knowledge of their spatial tuning properties to sinusoidal gratings. ${ }^{14}$

When the spatial Fourier components of the stimulus are considered and knowing that the amplitude of the VEP to the reversal of gratings is max- 


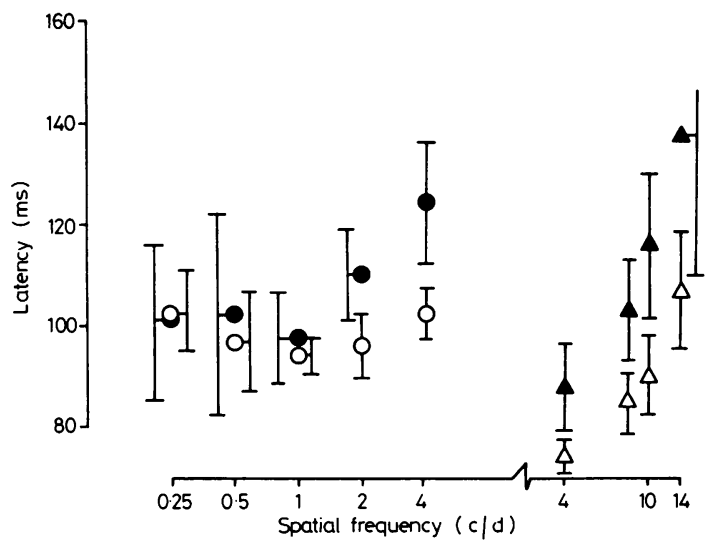

Fig 3 The mean latencies ( $\pm 1 S D$ ) of the P1 component to pattern reversal $(O, O)$ and the $N 1$ component to pattern onset $(\Delta, \Delta)$ are plotted. Open symbols give results obtained in 14 eyes of seven control subjects and closed symbols results obtained in 11 eyes affected by an attack of optic neuritis in the recent past. Significant latency differences are not seen below $2 \mathrm{c}^{\circ}$ and there is a tendency for delays to increase at higher spatial frequencies and to be greater in pattern onset than reversal responses.

imal at 2-4 $\mathrm{c}^{\circ 12}$ it may be predicted from DeValois et al' $\mathrm{s}^{14}$ findings that the VEP produced by a checkerboard having a periodicity of, say, $0.5 \mathrm{c} /{ }^{\circ}$ $\left(60^{\prime}\right.$ checks) would include a significant response to

$\circ$

$\circ$

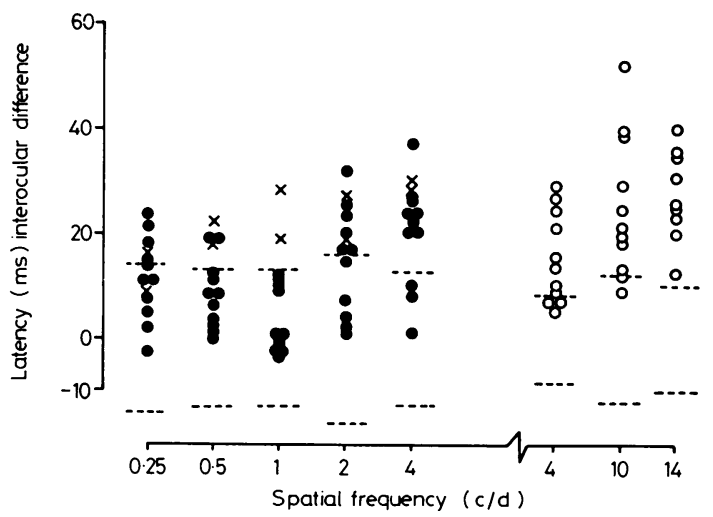

Fig 4 The data shown in fig 3 is here plotted in a different way. Individual differences in latency between the two eyes in the patients with unilateral optic neuritis are compared with the interocular latency difference in the seven control subjects $( \pm 3 S D)$. The peak latencies of the P1 to pattern reversal (O) and the NI to pattern onset (O) are shown. In two patients pattern onset responses could not be obtained (X). higher spatial harmonics in the region $2-4 \mathrm{c} /{ }^{\circ}$. The response to a checkerboard of $15^{\prime}$ checks, on the other hand, would be dominated by the response to its fundamental Fourier component, which is $\sqrt{ } 2$ times the periodicity of the checks and hence $3 \mathrm{c}^{\circ}$. In both instances, however, a similar population of cells is being stimulated. This is not to say that the checkerboard VEP will not show spatial selectivity. That it does so has been repeatedly demonstrated. ${ }^{15-17}$ The maximum VEP amplitude will be produced when the spatial Fourier component of the checkerboard having the greatest amplitude, its fundamental, coincides with the peak of the function relating VEP amplitude to the spatial frequency of sinusoidal gratings. This should occur with $15^{\prime}$ checks and, where the field size is comparable to that used in this laboratory, values close to this have often been found to give the maximum VEP amplitude. ${ }^{15} \quad{ }^{17-20}$

There is no comparable study employing a wide range of check size in individual cases of demyelinating optic nerve disease. Using steady-state stimulation Milner et al ${ }^{21}$ studied eight cases of optic neuritis and found all to have abnormal interocular latencies when a $50^{\prime}$ check size was used but only six to be outside the normal range using $14^{\prime}$ checks. Other investigations have not found significant or consistent changes in VEP delays with different check sizes. ${ }^{22}{ }^{23}$ For the reasons stated above, however, the author considers these results to be difficult to interpret with certainty.

In the present study, using gratings, a consistent pattern does seem to emerge. It was anticipated that a very high proportion of patients would have abnormal VEP latencies. Halliday et al ${ }^{1}$ found abnormalities in $90 \%$ of a similar group of patients as have subsequent investigators. ${ }^{321}$ Of interest in the results therefore is the lack of a significant delay when gratings of low spatial frequency are used and the greater delay found with pattern onset than with pattern reversal. There are two observations in the literature which may be of relevance to the former of these points. Firstly VEPs to unpatterned flash do not detect abnormalities as frequently as VEPs to patterned stimulation. ${ }^{123-25}$ Secondly, using uniform field flicker stimulation, Milner et al ${ }^{21}$ found delays in the medium temporal frequency range (13$25 \mathrm{~Hz}$ ) in all cases of optic neuritis but none of their subjects demonstrated delays when high temporal frequency stimulation was used $(35-60 \mathrm{~Hz})$.

This raises the possibility that the same neural elements are stimulated by high temporal frequency unpatterned flicker and low spatial frequency gratings and that these elements are less susceptible to the effects of demyelination. This may be because they are less likely to become demyelinated or, as 

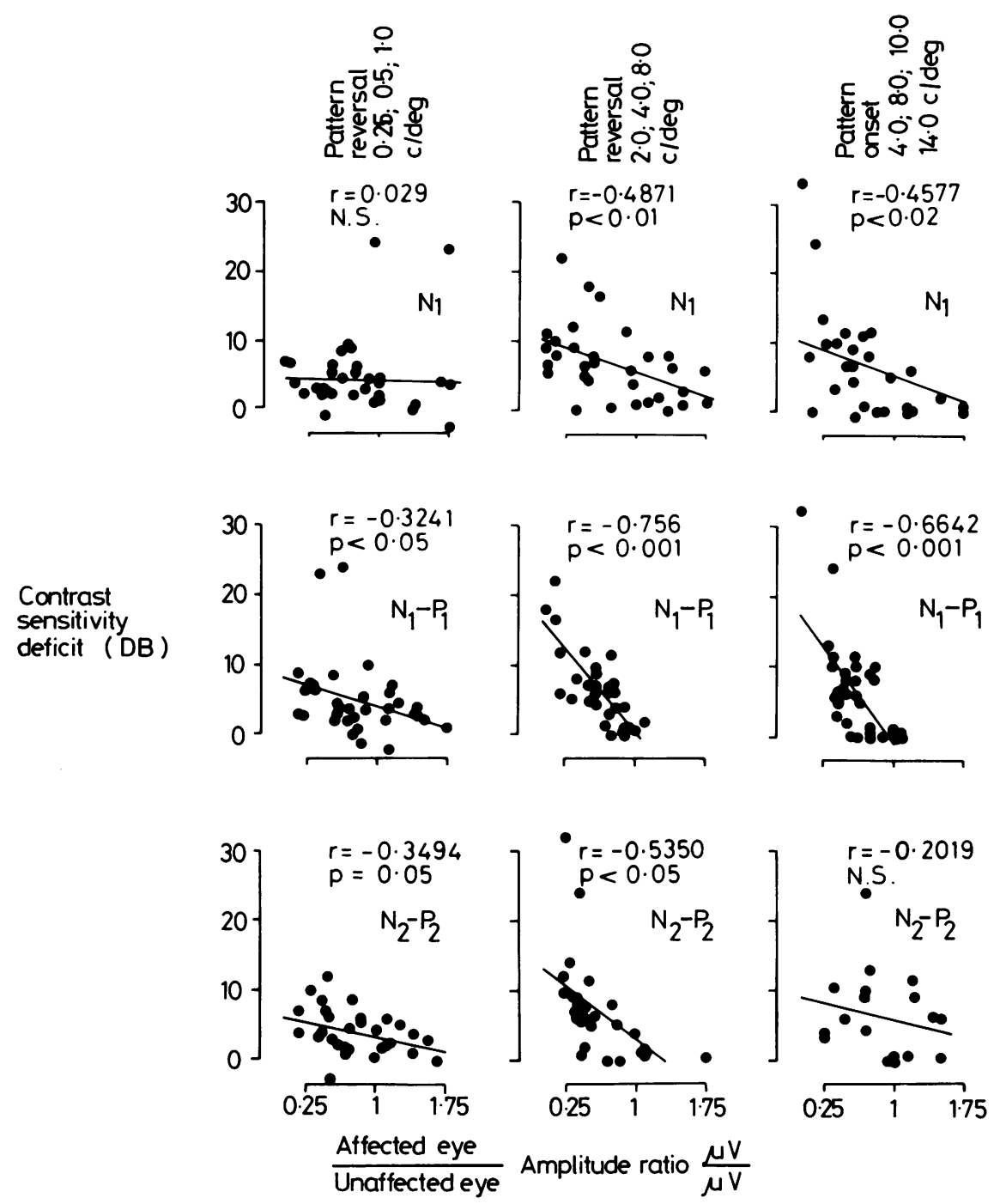

Fig 5 The ratio of the VEP amplitude between the affected and unaffected eyes of the patients is plotted against their contrast sensitivity deficit in $D B$ at the same spatial frequency. Results for pattern reversal above and below $1 \mathrm{c} /^{\circ}$ and for pattern onset are plotted separately. Only where a component could be clearly identified in the VEP from each eye has data been included, the N2 -P2 component was most frequently not present. Most of the components are related to the contrast sensitivity deficit but the correlation is strongest for $N 1-P 1$ above $1 \mathrm{c}^{\circ}$.

seems more likely, demyelination does not impair their function in the same way as other fibres. It is possible to offer an interpretation of this based on the " $X$ " and " $Y$ " classification originally described in retinal ganglion cells of the cat. ${ }^{26} \mathrm{Y}$ cells have larger receptive fields, larger diameter axons and faster axonal conduction velocities than $X$ cells and exhibit more transient responses to appropriate stimuli. Although the extent to which detailed correspondence between cat and human physiology in this respect can be inferred is uncertain ${ }^{27}$ there is reason to suppose that coarse gratings might predominantly, if not exclusively, activate transient mechanisms. ${ }^{28}$ Spekreijse ${ }^{29}$ furthermore, has argued that the different temporal frequency components of the flicker EP may reflect activity in the $X$ and $Y$ 
subsystems independently. Halliday ${ }^{30}$ has proposed a similar argument to explain the differences obtained using uniform-field flash and patternreversal checkerboard techniques. It may therefore be speculated that the Y system is less susceptible to the effects of optic nerve demyelination and this possibility is supported by widely differing evoked potential techniques.

Recent psychophysical investigations from this laboratory ${ }^{31}$ have demonstrated that threshold contrast sensitivity deficits found to low spatial frequency gratings in optic neuritis tend to diminish as the contrast of the grating is temporally modulated at higher rates, whereas in the case of medium spatial frequency gratings it remains constant. If it is the slower conduction velocities in demyelinated or remyelinated axons that results in the impairment of perception of stimuli undergoing rapid temporal modulation $^{32}$ then this psychophysical finding might be explicable in terms of the results shown in fig 3 . As Hess and Plant ${ }^{31}$ point out, however, sensitivity to low spatial frequency gratings modulated at high temporal rates is less affected by diminution in the area of the field (however this is achieved). Thus the occurrence of islands of preserved function offers an alternative or additional explanation. Further experiments are in progress to pursue this question.

As far as spatial frequencies above $1 \mathrm{c} /{ }^{\circ}$ are concerned a major point of interest is that pattern onset responses have longer delays than pattern reversal (fig 3 ) if the delay is expressed as a proportion of the expected normal latency. This finding lends itself to an interpretation which follows on from the discussion above. Pattern reversal responses above $3 \mathrm{c}^{\circ}$ may also be dominated by transient mechanisms but to a lesser extent as spatial frequency is increased. The argument and experimental evidence which favours this view have been discussed by Kulikowski. ${ }^{28}$ Pattern onset responses, on the other hand, may well consist principally of pattern specific mechanisms. That this might be identified with the $\mathrm{X}$ system is supported by the finding that pattern onset responses have a spatial selectivity higher in the spatial frequency spectrum than reversal responses, as $X$ cells are known to have smaller receptive field sizes. Thus it is possible that delays are greater with pattern onset than pattern reversal stimulation because a smaller proportion of the response reflects $Y$ cell activity. An alternative explanation is that the smaller axons of cells with smaller receptive fields are more susceptible to demyelination regardless of whether they are $\mathrm{X}$ or $\mathrm{Y}$, as within both classes there is a range of fibre diameters and conduction velocities. If so a gradual increase in the delay across spatial frequency would be expected rather than the observed differences between gratings above and below $1 \mathrm{c} /{ }^{\circ}$ and between onset and reversal responses.

Using a single check size Aminoff and Ochs ${ }^{33}$ found that pattern onset checkerboard VEPs detected abnormalities in a higher proportion of multiple sclerosis suspects than did pattern reversal. Also of relevance to the present results is the finding that the use of a small, square foveal stimulus was more useful in suspected MS than the pattern reversal checkerboard, ${ }^{8}$ as the use of a finer grating will similarly result in a greater foveal contribution to the response.

The extent to which the delayed VEPs observed in demyelinating optic nerve disease may be accounted for on the basis of decreased conduction velocity across demyelinated segments has been discussed by McDonald. ${ }^{34}$ On present evidence the conclusion is that it can only be a partial explanation. Nevertheless it is compatible with the finding in the present study that progressively greater delays are found as spatial frequency is increased above $1 \mathrm{c}^{\circ}$ for both reversal and onset VEPs. If it is assumed that the conduction velocity across a demyelinated segment is diminished by a constant factor (25 times according to McDonald's calculations) then the slower conducting the group of fibres, the longer will be the delay.

It is of interest to consider the present results in the light of the theory of visual processing which suggests in part a quasi-Fourier analysis of the visual image implying co-operation between spatial channels of restricted bandwidth. ${ }^{35}{ }^{36}$ If the increase in latency of VEPs to gratings of higher spatial frequency (paralleled by an increase in reaction time ${ }^{37}$ ) reflects differences in retino-cortical conduction time then in the pathological situation, however, if a different added delay is produced in different spatial frequency channels there might be impairment of the visual system's ability to discriminate phase relationships. This would not result in a severely degraded image ${ }^{38}$ but might lead to subtle abnormalities of visual perception as has been suggested may operate in amblyopia. ${ }^{39}$

For a number of reasons it was anticipated that a correlation would be found between VEP amplitude and contrast sensitivity anomalies: Halliday et al $^{40}$ found that the amplitude of the checkerboard VEP in optic neuritis is related to Snellen acuity (although this was not found by others in multiple sclerosis $^{3}$ ) and it is known that threshold contrast sensitivity deficits in optic neuritis persist in suprathreshold conditions. ${ }^{41}$ Furthermore VEP amplitude is linearly related to log contrast and it is possible to predict threshold contrast sensitivity by extrapolation to zero voltage. A strong correlation, however, was only found for the N1-P1 component and only 
above $1 \mathrm{c} /^{\circ}$. Although (as outlined in the results section) this may in part be explicable on the basis of the greater scatter of the other parameters measured, it raises the possibility that only in the case of the N1-P1 component and only above $1 \mathrm{c} /{ }^{\circ}$ are the same neural elements being studied by the evoked potential and threshold contrast sensitivity techniques. The results at low spatial frequencies might also be related to Sjörstrand et al' $\mathrm{s}^{41}$ finding that the perception of coarse gratings in suprathreshold conditions is not as simply related to threshold sensitivity as is the perception of higher spatial frequency gratings.

Contrast sensitivity loss in this group of patients did not vary uniformly across spatial frequency, confirming the findings of other investigators. ${ }^{42}{ }^{43}$ Latency changes, on the other hand, do appear to vary in a consistent manner. It is therefore not anticipated that a relationship between threshold contrast sensitivity and latency would be found. Bodis-Wollner et $\mathrm{l}^{44}$ failed to demonstrate such a correlation when comparing threshold losses and grating VEP latency in a large number of patients but at a single spatial frequency $\left(2 \cdot 3 \mathrm{c} /{ }^{\circ}\right)$.

How might the present results be applied to the task of designing an optimum VEP stimulus to detect subclinical demyelination in suspected multiple sclerosis? Clearly as high a spatial frequency as possible should be employed and in this respect the pattern onset response may well prove advantageous. A coarser pattern, however, should be reserved for patients with impaired central vision.

The author is grateful to the Wellcome Trust of Great Britain for support. Thanks are also due to Drs MFT Yealland and IMS Wilkinson for permission to study patients under their care and to the staff of the EEG Department of Addenbrooke's Hospital for technical assistance. Professor FW Campbell has provided invaluable advice and encouragement.

\section{References}

' Halliday AM, McDonald WI, Mushin J. Delayed visual evoked response in optic neuritis. Lancet 1972;i:982-5.

${ }^{2}$ Halliday AM, McDonald WI, Mushin J. Visual evoked response in the diagnosis of multiple sclerosis. $\mathrm{Br} \mathrm{Med}$ J 1973;4:661-4.

${ }^{3}$ Asselman P, Chadwick DW, Marsden CD. Visual evoked response in the diagnosis and management of multiple sclerosis. Brain 1975;98:261-82.

${ }^{4}$ Matthews WB, Small DG, Small M, Pountney E. Pattern reversal evoked visual potential in the diagnosis of multiple sclerosis. J Neurol Neurosurg Psychiatry
1977;40:1009-14.

${ }^{5}$ Cant BR, Hume AL, Shaw NA. Effect of luminance on the pattern visual evoked potential in multiple sclerosis. Electroencephalogr Clin Neurophysiol 1978;45:496-504.

- Camisa J, Leland HM, Bodis-Wollner I. The effect of stimulus orientation on the visual evoked potential in multiple sclerosis. Ann Neurol 1981;9:532-9.

${ }^{7}$ Coupland SG, Kirkham TH. Orientation-specific visual evoked potential deficits in multiple sclerosis. Can $J$ Neurol Sci 1982;9:331-7.

${ }^{8}$ Hennerici M, Wenzel D, Freund H-J. The comparison of small-size rectangle and checkerboard stimulation for the evaluation of delayed visual evoked responses in patients suspected of multiple sclerosis. Brain 1977;100:119-36.

${ }^{9}$ Celesia GG, Daly RF. Visual electroencephalographic computer analysis, (VECA). Neurology (Minneap) 1977;27:637-41.

${ }^{10}$ Schade OH. Optical and photoelectric analogue of the eye. J Opt Soc Am 1956;46:721-39.

"Campbell FW, Robson JG. Application of Fourier analysis to the visibility of gratings. J Physiol (Lond) 1968;197:551-6.

12 Plant GT, Zimmern RL, Durden K. Transient visually evoked potentials to the onset and pattern reversal of sinusoidal gratings. Electroencephalogr Clin Neurophysiol 1983;56:147-58.

${ }^{13}$ Maffei L, Fiorentini A. The visual cortex as a spatial frequency analyser. Vision Res 1973;13:1255-67.

${ }^{14}$ DeValois KK, DeValois RL, Yund EW. Response of striate cortex cells to gratings and checkerboard patterns J Physiol (Lond) 1979;291:483-505.

${ }^{15}$ Spekreijse H. Analsis of EEG responses in man evoked by sine wave modulated light. Thesis: DW Junk, The Hague 1966:161.

${ }^{16}$ Harter MR. Evoked cortical potentials to checkerboard patterns: effect of check size as a function of retinal eccentricity. Vision Res 1970;10:1365-76.

${ }^{17}$ Regan D, Richards W. Independence of evoked potentials and apparent size. Vision Res 1971;11:679-84.

${ }^{18}$ Bartl G, Van Lith GHM, Van Marle GW. Cortical potentials evoked by a TV pattern reversal stimulus with varying check size and stimulus field. $\mathrm{Br} \mathrm{J} \mathrm{Oph-}$ thalmol 1978;62:216-9.

${ }^{19}$ Arden GB, Faulkner DJ, Mair C. A versatile TV pattern generator for visual evoked potentials. In: Desmedt JE, ed. Visual Evoked Potentials in Man: New Developments. Oxford: Clarendon Press, 1977: 91-109.

${ }^{20}$ Sokol S. Visual evoked potentials to checkerboard patterned stimuli in strabismic amblyopia. In: Desmedt JE, ed. Visual Evoked Potentials in Man: New' Developments. Oxford: Clarendon Press, 1977:411-7.

${ }^{21}$ Milner BA, Regan D, Heron JR. Differential diagnosis of multiple sclerosis by visual evoked potential recording. Brain 1974;97:755-72.

${ }^{22}$ Cook JH, Arden GB. Unilateral retrobulbar neuritis: a comparison of evoked potential and psychological measurements. In: Desmedt JE, ed. Visual Evoked Potentials in Man: New Developments. Oxford: Clarendon Press, 1977:451-7. 
${ }^{23}$ Duwaer AL, Spekreijse $H$. Latency of luminance and contrast evoked potentials in multiple sclerosis patients. Electroencephalogr Clin Neurophysiol 1978;45:244-58.

${ }^{24}$ Rouher F, Plane C, Solé P. Interêt des potentials évoqués visuels dans les affections du nerf optique. Archs Ophthalmol Rev Gen Ophthalmol 1969;29:555-64.

${ }^{25}$ Richey ET, Kooi KA, Tourtellotte WW. Visually evoked responses in multiple sclerosis. J Neurol Neurosurg Psychiatry 1971;34:275-80.

${ }^{26}$ Enroth-Cugell C, Robson JG. The contrast sensitivity of retinal ganglion cells of the cat. J Physiol (Lond) 1966;187:517-52.

${ }^{27}$ Lennie P. Parallel visual pathways. Vision Res 1980;20:561-94.

${ }^{28}$ Kulikowski JJ. Separation of occipital potentials related to the detection of pattern and movement. In: Desmedt JE, ed. Visual Evoked Potentials in Man: New Developments. Oxford: Clarendon Press, 1977: 184-96.

${ }^{29}$ Spekreijse H. Pattern evoked potentials: principles, methodology and phenomenology. In: Barber C, ed. Evoked Potentials. Lancaster MTP Press, 1980:5573.

${ }^{30}$ Halliday AM. Visual Evoked Potentials in Demyelinating Disease: Basic and Clinical Electrophysiology. New York: Raven Press, 1981:201-15.

${ }^{31}$ Hess RF, Plant GT. The effect of temporal frequency variation on threshold contrast sensitivity deficits in optic neuritis. J Neurol Neurosurg Psychiatry 1983;46:322-30.

${ }^{32}$ McDonald WI, Sears TA. The effects of experimental demyelination on conduction in the central nervous system. Brain 1970;93:583-98.

${ }^{33}$ Aminoff MJ, Ochs AL. Pattern onset visual evoked potentials in suspected multiple sclerosis. J Neurol Neurosurg Psychiatry 1981;44:608-14.
${ }^{34}$ McDonald WI. Pathophysiology of conduction in central nerve fibres. In: Desmedt JE, ed. Visual Evoked Potentials in Man: New Developments. Oxford: Clarendon Press, 1977:427-37.

${ }^{35}$ Blakemore CB, Campbell FW. On the existence of neurones in the human visual system sensitive to the orientation and size of retinal images. $J$ Physiol (Lond) 1969;203:237-60.

${ }^{36}$ Georgeson MA. Spatial frequency analysis in early visual processing. Phil Trans $R$ Soc B 1980;290:11-22.

${ }^{37}$ Bretmeyer BG. Simple reaction time as a measure of the temporal response of transient and sustained channels. Vision Res 1975;15:1411-4.

${ }^{38}$ Piotrowski LN, Campbell FW. A demonstration of the visual importance and flexibility of spatial frequency amplitude and phase. Perception 1982;11:337-46.

${ }^{39}$ Hess RF, Burr DC, Campbell FW. A preliminary investigation of neural function and dysfunction in amblyopia. III. Co-operative activity of amblyopic channels. Vision Res 1980;20:757-60.

${ }^{40}$ Halliday AM, McDonald WI, Mushin J. Delayed pattern visual evoked responses in optic neuritis in relation to visual acuity. Trans Ophthalmol Soc UK 1973;93:315-24.

${ }^{41}$ Sjöstrand S, Abrahamsson M. Suprathreshold vision in acute optic neuritis. J Neurol Neurosurg Psychiatry 1982;45:227-34.

${ }^{42}$ Regan D, Silver R, Murray TJ. Visual acuity and contrast sensitivity in multiple sclerosis: hidden visual loss. Brain 1977;100:563-73.

${ }^{43}$ Zimmern RL, Campbell FW, Wilkinson IMS. Subtle disturbances of vision after optic neuritis elicited by studying contrast sensitivity. J Neurol Neurosurg Psychiatry 1979;42:407-12.

${ }^{44}$ Bodis-Wollner I, Hendley CD, Mylin LH, Thornton J. Visual evoked potentials and the visuogram in multiple sclerosis. Ann Neurol 1979;5:40-7. 